This item was submitted to Loughborough's Research Repository by the author.

Items in Figshare are protected by copyright, with all rights reserved, unless otherwise indicated.

\title{
Mathematical relativism: logic, grammar, and arithmetic in cultural comparison
}

PLEASE CITE THE PUBLISHED VERSION

http://dx.doi.org/10.1111/j.1468-5914.2006.00299.x

PUBLISHER

(C) The Executive Management Committee / Blackwell Publishing

VERSION

AM (Accepted Manuscript)

LICENCE

CC BY-NC-ND 4.0

REPOSITORY RECORD

Greiffenhagen, Christian, and Wes Sharrock. 2019. "Mathematical Relativism: Logic, Grammar, and Arithmetic in Cultural Comparison". figshare. https://hdl.handle.net/2134/14205. 
This item was submitted to Loughborough's Institutional Repository (https://dspace.lboro.ac.uk/) by the author and is made available under the following Creative Commons Licence conditions.

\section{creative
commons}

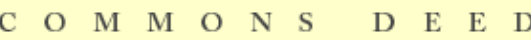

Attribution-NonCommercial-NoDerivs 2.5

You are free:

- to copy, distribute, display, and perform the work

Under the following conditions:

Attribution. You must attribute the work in the manner specified b the author or licensor.

Noncommercial. You may not use this work for commercial purposes.

No Derivative Works. You may not alter, transform, or build upon this work.

- For any reuse or distribution, you must make clear to others the license terms of this work.

- Any of these conditions can be waived if you get permission from the copyright holder.

Your fair use and other rights are in no way affected by the above.

This is a human-readable summary of the Leqal Code (the full license).

\section{Disclaimer 만}

For the full text of this licence, please go to: http://creativecommons.org/licenses/by-nc-nd/2.5/ 


\section{Mathematical Relativism:}

\section{logic, grammar, and arithmetic in cultural comparison}

Authors: Christian Greiffenhagen and Wes Sharrock

\section{Address:}

Christian Greiffenhagen

Department of Sociology

Roscoe Building

University of Manchester

Manchester M13 9PL

UK

Tel: +44 1612752497

Fax: +44 1612752514

Email: Christian.Greiffenhagen[replace_with_@]manchester.ac.uk

Version: March 8, 2006 


\title{
Mathematical Relativism:
}

\section{logic, grammar, and arithmetic in cultural comparison}

\author{
Abstract \\ Cultural relativism is supposed to be a bold and provocative thesis. In this paper we \\ challenge the idea that it is an empirical thesis, i.e., one that is supported through \\ anthropological and historical examples. We focus on mathematical relativism, the \\ view that a mathematics from another culture or time might be so radically divergent \\ from our mathematics that 'theirs' would stand in direct conflict with 'ours' (and in \\ that sense constitute an alternative mathematics).
}

We question in what sense the examples given to support the general thesis are relativistic about mathematics and argue that on close inspection they are not, and certainly not in any radical sense. We do not contest the fact that there can be great mathematical diversity between cultures, but wonder whether it makes sense to talk of ‘the same’ mathematical forms in heterogeneous mathematical environments. Finally, while relativists see the later Wittgenstein as providing support for their own thesis, we claim that Wittgenstein argues against both realism and relativism. cultural comparison. Journal for the Theory of Social Behaviour 36 (2), 97-117. 


\section{Introduction}

This article is part of a critical analysis of the way empirical examples feature in arguments for cultural relativism, which we take to be the thesis that logic, grammar, and mathematics are, in some sense, only locally valid ${ }^{1}$. Our interest lies in pinning down just what 'in some sense' might amount to. In companion papers (Greiffenhagen and Sharrock, forthcoming a,b) we deal with logical and linguistic relativism, while in this article we discuss a variety of examples of different forms of mathematical relativism - for example Watson (1987, 1990), Verran (2000a, 2000b, 2001), Bloor (1973, 1976, 1994), Jennings (1988), Raven (1996), and Reed and Lave (1979). We are aware that these examples only fall uneasily under one umbrella term. As Louch (2000 [1966], p.247) remarks:

cultural relativism is less a single doctrine or position than a collection of them. A number of different and logically unconnected views have been put forth under this label.

What we are concerned with is the way in which the sociological or historical examples are presented and the way in which conclusions are drawn from them. In our view, the conclusions are typically not sustained by the offered evidence and alternative characterizations of it are at least as plausible. That is to say, we question in what sense these studies are relativistic about mathematics and argue that 'relativism' is a misnomer for instances of cultural heterogeneity.

\footnotetext{
${ }^{1}$ There are yet other variations in the cultural relativist's use of evidence, such as those of cultural psychology, which are addressed in a related paper (Greiffenhagen, forthcoming).
}

Greiffenhagen, C. and W. Sharrock (2006). Mathematical relativism: logic, grammar, and arithmetic in cultural comparison. Journal for the Theory of Social Behaviour 36 (2), 97-117. 
The thing that seems most threatening about relativism is its implication (not necessarily overtly advocated) that cultural differences of numbers or counting practices carry ontological consequences. Mathematical relativism, by saying that mathematics is not universally but only locally true, seems to subvert realists' or idealists' claims about the generality of mathematics and about the stability of material nature. According to the relativistic thesis it is possible that there are cultures which not only have a patently different form of mathematical practice from ours, but where within that practice what 'we' take for a mathematical truth is directly contradicted: 'here' two oranges added to five oranges would give us seven oranges, but 'there' it would give us eleven of them.

We doubt that there can be the radically different mathematics that relativists envisage, i.e., aspects of mathematics that are conceptually or ontologically confrontational with ours. However, we are not denying that there are many cultural variations in numbers and counting practices - or that some of them might be hard for us to make sense of ${ }^{2}$. To give a few examples: the French call 80 "quatre-vingt" (which could be 'represented' as $4 \times 20)$ while the English call it “eighty” $(8 \times 10)$; while the English group three digits together (thousands) the Chinese group four digits together (ten thousands, wan in Chinese), hence "fifty thousand" $(50 \times 1,000)$ in English but "wu wan" $(5 \times 10,000)$ in Chinese; and the Oksapmin have devised a bodycounting system up to twenty-seven, rather than the Western finger-counting up to ten

\footnotetext{
${ }^{2}$ See, for example, Baker and Hacker’s (1985, pp. 323-325) description of a traditional Japanese system for telling the time.
}

Greiffenhagen, C. and W. Sharrock (2006). Mathematical relativism: logic, grammar, and arithmetic in cultural comparison. Journal for the Theory of Social Behaviour 36 (2), 97-117. 
(e.g., Saxe, 1982; Saxe and Moylan, 1982). These (and many, many more) are examples of cultural variation in mathematical practices. However, they are despite their differences also readily recognizable as diverse forms of mathematics and relatively easy to translate, coordinate, and learn. In contrast, for relativists these kinds of differences seem to exhibit an ontological divergence, as they raise questions about what numbers and mathematics 'are', e.g.:

[this study] raises the challenging question of what mathematics and logics are in the contexts of working across cultures. (Verran, 2000a, p.58)

My contention is that 'natural number' is a cultural construct, differently formulated in different societies. (Watson, 1990, p.283)

Many of the examples that we discuss in this paper explicitly draw upon the later Wittgenstein. We also approach these questions from a Wittgensteinian perspective, but draw very different implications from his writings. We agree with relativists that Wittgenstein's writings contain a sustained attack on realist theories. However, relativists the relativist reading of Wittgenstein (e.g. Bloor, 1973, 1976) sees Wittgenstein as developing an alternative (relativistic) theory of social life - a theory that could, in their view, be improved and advanced through a more careful presentation of 'empirical' historical or sociological examples. In contrast, we see Wittgenstein as not only attack realism, but also attacking relativism - by questioning the coherence and sense of their respective claims. In our view, Wittgenstein argues that before we can ask whether a certain theory is true or false, we need to explore whether the theory says anything intelligible. 


\section{An exemplary case: Mr. Ojo}

We will try to show that the kind of relativist project we are debating here is based on two assumptions:

(1) that being able to do sums requires the possession of an ontology about what numbers are (i.e., what they stand for or how they can connect with things in the world);

(2) that this ontology is of the same form that foundationalist philosophers of science and mathematics (e.g., von Neumann, Zermelo, Frege, or Carnap) engage in (as though one of two equivalent formalizations could more closely match the understanding that ordinary users have of everyday arithmetic).

We begin by exhibiting these two assumptions in Helen Verran's ${ }^{3}$ prize-winning treatment of teaching arithmetic in Western and Yoruba schools (Watson, 1987, 1990; Verran, 2000a,b, 2001). In our view, Verran’s renderings exaggerate the divergence between two mathematically equivalent ways of measuring length, both of which are comfortably contained within 'our' Western arithmetic. Her aim is to demonstrate a substantial cultural difference in 'concepts of length', but she thereby disregards the fact that the two methods presuppose the same specification of an individual's height and only vary in the ways in which that height is determined.

\footnotetext{
${ }^{3}$ Helen Verran used to publish as Helen Watson. We will throughout refer to her as 'Verran', citing, where necessary, her earlier papers as 'Watson'.

Greiffenhagen, C. and W. Sharrock (2006). Mathematical relativism: logic, grammar, and arithmetic in cultural comparison. Journal for the Theory of Social Behaviour 36 (2), 97-117.
} 
Verran presents the case of a Yoruba teacher, Mr. Ojo, whose teaching style is contrasted with a Western way of teaching pupils how to measure people's heights. The Western teaching method is explained thus:

In the lab we had measured each other: we used the string to represent height, lay the string on the floor and used chalk to record the length. Then when one of the few metre rulers became available, we measured the distance between chalk marks and recorded the measurement in a chart (Verran 2000a, p. 59).

Rather than doing the same, Mr. Ojo used another procedure, which also involved one piece of string. However, instead of the meter ruler Mr. Ojo used a card of $10 \mathrm{~cm}$ length with which he measured the string:

He called a small boy to the front: with the end of string just under the boy's heel, he held his finger at the point on the string which matched the top of the boy's head. Tying a loose knot at this point, he took the other end of the string from under the boy's foot; holding this at one end of a card he wound the length of string around until he came to the knot. Then he instructed: 'Count the number of strings around the card, e.g., "9" (i.e., $10 \mathrm{~cm}$ lengths) and write down the number. Multiply by ten. How do we multiply by ten? ... ninety ... now add the bit of string left over ... Yes, we have $96 \mathrm{~cm}$.' (p.60)

Verran then claims that "Mr Ojo had deviated significantly from the exemplary lesson" (p. 60; our emphasis). The question for us is to figure out what this significant difference might be.

In both cases a piece of string is held against a pupil's body to capture the height of the pupil. The difference lies in the method used to measure this piece of string. In the first case, the height is stored on the floor by using chalk marks to record the cultural comparison. Journal for the Theory of Social Behaviour 36 (2), 97-117. 
captured height. These marks are subsequently measured using a meter ruler. In the second case, the piece of string is wrapped around a piece of card of known length. Consequently, the number of wrappings and the leftover can be used to calculate the height.

Verran does not claim that the two methods measure different heights. In other words, mathematically the two methods are equivalent, as children who learn the former technique and children who learn the latter would not arrive at different numerical determinations of height (in fact, children who have learned basic computational operations should be able to understand both procedures). The difference lies in the instrument used to measure, namely a meter ruler and a card respectively.

For Verran, the different methods based on these different instruments are supposed to teach different 'ontologies' of height. In other words, because the teaching methods are different, the children are supposed to have acquired a different understanding of the nature of height. For Verran (2000a, p. 60) this significant difference is the result of substituting "the prescribed stretched string and the extending metre" with "a small card wound around with string”:

Disconcertingly he [Mr. Ojo] had measurement of length beginning in the plurality of strands of wound-up string rather than in the singularity of an extension. Should he have been corrected? (p.60; our emphasis).

Or elsewhere: 
I was scandalized. Mr. Ojo was presenting a bundle of short strands of string, a plurality, as length instead of demonstrating the prescribed singular extension. [...] I was profoundly confused and puzzled. (Verran, 2001, p.3)

Verran thinks that she has observed the teaching of two contrasting (ontological) concepts of length, on the one hand, as a "plurality of strands of wound-up string" ('Yoruba'), and, on the other, as "the singularity of an extension” ('Western'). Verran here exhibits the first assumption above, namely that the teaching of the concept of length is supposed to involve more than learning how to determine the length of a certain item. Verran thinks that apart from being taught how to measure the length of things, pupils are also taught something else, something deeper - namely an understanding of the essential nature of length.

Verran brushes over the mathematical equivalence of the two practices of measuring and instead stipulates that they imply that a different ontological concept of length is being taught. As this difference is not directly visible in the results of measurements (which are the same), Verran in effect interprets the concept of length in a form of operationalism, i.e., as though a single procedure of measuring something could constitute and fully exhaust the concept of length. For Verran it seems that each concept of length will allow only one way of measuring it. As with many others discussing everyday arithmetic, she thereby overlooks the interoperability between diverse measurement techniques (the concept of length surely involves understanding its constancy across different modes of determination). In other words, Verran neglects the extent to which we often have a variety of alternative and equivalent practices available to arrive at the same result (as anyone who has ever tried to cultural comparison. Journal for the Theory of Social Behaviour 36 (2), 97-117. 
measure domestic space using whatever domestic objects or body parts are available will know).

Nowhere does Verran demonstrate that the supposed difference between the 'Western' and 'Yoruba' concepts ever becomes visible in everyday computations ${ }^{4}$. Furthermore, she neglects the blatant similarities between the two methods of measurement, in particular the role of the piece of string which in both cases is initially used to capture the pupil's height (by singular extension). Even in Mr. Ojo’s method (that is supposed to teach a concept of length as a "plurality of strands of wound-up string”) the starting point is to represent height as "the singularity of an extension”: Mr. Ojo’s operation first gives the string a singular extension (from head to foot of the child being measured) and then uses the card to measure the string. Note that this does not involve a contrast between two conceptions of length, once as a singular "the singularity of an extension" (the straight line) and the other time as "a plurality of wound-up strings" (the string wrapped around the card), but instead involves using the card to calculate the length of the straight line (which is done by treating the card as a singular extension, and the string to represent a simple multiple of a number of shorter but equivalent straight line segments, plus a remainder).

Verran then goes on to exhibit the second assumption mentioned above: in order to express the supposed difference between the Yoruba and the Western concept of length, she invokes a philosopher of science, namely Rudolf Carnap and his Philosophical Foundations of Physics (1966). Verran argues that for Carnap length is

\footnotetext{
${ }^{4}$ In other words, the ‘difference’ is very much like Wittgenstein’s (1953, §293) beetle in the box.
} cultural comparison. Journal for the Theory of Social Behaviour 36 (2), 97-117. 
a quantitative concept that captures "universal qualities in [...] spatio-temporal entities” (Verran, 2000a, p.63). We do not defend Carnap’s logical positivism, but are surprised that it has any implications for the case under discussion. However, Verran claims:

According to [Carnap's] account of logic and mathematics, Mr Ojo is wrong to present an image to the children where length is portrayed as a bundle of string whose many strands can be counted and manipulated to come up with a single value for length. An uninterrupted straight line, sectioned in a subsequent action, portrays the proper and only correct way to do length. (p.64)

According to our understanding of Carnap the only bearing that his philosophy could have on this example is the claim that each object will have a unique and definite length. Carnap is concerned with length as a property of objects and for him it is a requirement for such a quantitative concept that it will possess a single determination for any object. In other words, for Carnap it is entirely conceivable that we might have different techniques to measure length, but it is a requirement that all of these techniques will produce the same result.

As already mentioned, this is exactly what we witness in the case under discussion: both measuring techniques are, mathematically, equivalent. Consequently, we cannot imagine any grounds upon which Carnap would have objected to Mr. Ojo’s lesson. In other words, Carnap would recognize that Mr. Ojo is teaching children how to measure correctly, which in this context means arriving at the appropriate results (using measurement in conjunction with calculation, rather than just measurement). cultural comparison. Journal for the Theory of Social Behaviour 36 (2), 97-117. 
Verran here exhibits the relativist's tendency to conflate length with philosophical reflections about the nature of length. However, philosophical disagreements about what length 'is’ are not enough to demonstrate that people’s concepts of length (i.e., their understanding of how length is to be measured or calculated) is different.

\section{The universal character of mathematics}

After this long analysis of one example let us return to the general aim of mathematical relativism, which is to contest the claim that arithmetic and mathematics are 'universal'. In this section we therefore want to review what the universal character of mathematics might amount to.

The practices of our standard arithmetic (i.e., base ten, Arabic numerals, grouping thousands together, etc.) are invariant. They are to be done everywhere the same, with no room for variation. Given that standard arithmetic is being done (i.e., that we are operating within this system), then calculations must be done in ways that satisfy these rules and must yield these results, regardless of who is doing it.

However, standard arithmetic only exercises its compelling and inexorable character when one is operating in terms of it. Standard arithmetic, of itself, does not compel anyone to use it. Of course, there might be practical reasons for using it: the educational system and the extensive institutionalization of its practice might impose on people the practical necessity to learn and use it (if you want to know how much money the cash machine will let you have, you had better be able to use arithmetic; or, in government policy terms: if you want to get a good job in a sophisticated, well 
paying form of modern employment, you had better learn arithmetic). However, nothing 'in' the system compels people to use this system.

Furthermore, standard arithmetic does not compel all people to calculate in specific ways. There are various ways available for doing calculations (e.g., in your head, with your fingers, or using pen and paper) just as there are variable computational forms for arriving at the same result (e.g., $23 \times 12=230+46=276$ or $23 \times 12=240+36=$ 276). There is no reason to suppose that all those who are doing arithmetic must proceed in the same specific ways in working through a computation, only that those doing standard arithmetic must arrive at the same results.

Is 'our' arithmetic then universal? To cast the question in something like the Enlightenment idea: Would all rational beings be compelled to accept 'our' standard arithmetic? As Wittgenstein would put it: the question contains a mistake. Standard arithmetic is not something that we in Western societies 'accept'. It is therefore not something we accept on rational grounds either (neither do we accept it 'irrationally'). We simply need no grounds to accept the arithmetic we are taught, since this is the arithmetic that we are being taught ${ }^{5}$. However, saying that we are taught this arithmetic does not mean that

(a) there might not be different ways to solve an arithmetic problem;

\footnotetext{
${ }^{5}$ Imagine a society where children are taught two different arithmetics and then, at a certain age, would have to 'choose' between them.

Greiffenhagen, C. and W. Sharrock (2006). Mathematical relativism: logic, grammar, and arithmetic in cultural comparison. Journal for the Theory of Social Behaviour 36 (2), 97-117.
} 
(b) that we are thereby being taught that this is the only kind of arithmetic there can possibly be;

(c) that we are taught arithmetic as something for whose correctness there is evidence and justification that can be given to us.

From a very early age children are rigorously rehearsed in numeracy practices. However, they are not provided with theoretical explanations of them (e.g., historicalphilosophical accounts of why we count with base ten rather than base eight). Learning arithmetic is a matter of learning to replicate what other competent users of it do. What one is being taught is how to use the arithmetical system correctly - not why it is correct or what makes it correct. In other words, contra relativists such as Jennings (1988, p.100) who want to say that we 'believe' in $5+7=12$, we would argue that arithmetic is not something that anyone believes in $^{6}$. As Wittgenstein (1978) puts it:

But I feel a temptation to say: one can't believe that $13 \times 13=196$, one can only accept this number mechanically from somebody else. (I §106, p. 76)

For I want to say: "One can only see that $13 \times 13=196$, and even that one can't believe. And one can - more or less blindly - accept a rule.” (I §109, p. 77)

We engage in our arithmetic rather than, say, an aboriginal way of counting, simply because that is the arithmetic that we have been taught. This does not mean that we

\footnotetext{
${ }^{6}$ Neither do we drive on the left because we 'believe' in it - although one might say that we believe in the existence of a Big Bang.

Greiffenhagen, C. and W. Sharrock (2006). Mathematical relativism: logic, grammar, and arithmetic in cultural comparison. Journal for the Theory of Social Behaviour 36 (2), 97-117.
} 
therefore 'disbelieve' other ways of counting. We engage in ours pretty much because it is ours, and they in theirs because it is theirs. This includes, of course, all kinds of practical reasons why we would not want to switch. All kinds of disadvantages for us would attach to adopting their system (not least because all kinds of infrastructural practices in our society have our form of numeracy built in) and most of ours would be useless to them.

\section{The social/cultural character of mathematics}

It is no great surprise to us that other cultures have different mathematics than we do (though the kind of mathematics they have might be somewhat surprising to our mathematicians). In fact, would it not seem much more surprising if anthropologists had found cultures which, despite their enormous organizational differences and complete cultural autonomy from us, had developed a mathematics thoroughgoingly identical to ours? Wouldn't that seem like the most astonishing thing?

However, accepting this does not mean that there can be infinite variation between cultures in respect of what mathematics can be. We do not say this because we hold some realist doctrine about the way in which the nature of things imposes itself upon our thought to limit what a mathematics can be, but because of the role translation plays: if some other culture's supposed mathematical practice is barely recognizable as such to us, then why have would we identify it as 'mathematics'? Without some kinship between our and their practice there would seem little point in treating them 
as playing equivalent parts in the respective cultures ${ }^{7}$. We might as well conclude that these people lack a mathematics, and have instead some other practice that our culture does not feature ${ }^{8}$ :

If you have two different ways of multiplying, why call them both multiplying? Why not call one multiplying and the other dividing, or one multiplying-A and the other multiplying-B, or any damn thing? It is simply that you have two different kinds of calculations and you have not noticed that they give different results. (Wittgenstein, 1976, p. 216)

In other words, in order to translate an alien practice as 'mathematics', there must be some significant similarity to our practice of mathematics. Otherwise, we might find a new way of translating that alien practice (as, e.g., karate, feng-shui, or raindancing). It is only because the alien practice resembles our practice of mathematics that we identify it in translation as mathematics. This is not because we assume our language has some privileged universality, but because this is how speak in our language of what 'they' do:

Money has a special role in our lives, and this is what justifies translating foreign terms as 'money'. Mathematics has a special

\footnotetext{
${ }^{7}$ The kinship should not be thought of in terms of any specific notation, procedure, or method, but as one to be identified with respect to the part that the relevant activity plays in their lives. However odd a possible way of totalling up might seem to us, if doing things that way fits into, e.g., buying and selling goods in a similar way as it does in ours, then it may well rightly be identified as a way of totalling up.

${ }^{8}$ To avoid the imputation of ethnocentrism, of placing 'our' mathematics in a privileged place which determines what is 'really' mathematics and what is not, we should perhaps say that our point is more of the order of: if it doesn't look like a duck, doesn’t walk like a duck, doesn't sound like a duck, then why on earth are you determined to call it a duck? Ethnocentricism comes with a misplaced pride in 'our' possession of mathematics, such that it would seem a negative point about a culture that it had no mathematics, but there is no need to read any such devaluation into the fact that another culture does not have the same configuration of institutions we do, however proud of these institutions some of 'us' might be.
} 
role in our lives and special characteristics, and these are what justify calling alien practices ‘mathematics'. (Gerrard, 1991, p.139)

Given that a culture has a practice that could be identified as mathematics, then we may reasonably expect that its basic forms ${ }^{9}$ will be extensively standardized amongst the members of that society. We do not for one moment expect to find that a culture features 'mixed mode' mathematics, where the distribution of counting practices amongst them was arbitrarily, even randomly assorted. We just would not call such a mixed mode practice 'mathematics' (but perhaps guessing, playing, or consulting an oracle).

We also assume that the kind of mathematics that might have been created in a community will be the collective product of the work of a group of mathematicians, rather than any single mathematician (whether or not there exists a specific profession of mathematicians or mathematics has been developed as part of other activities, e.g., accounting or religion). We also assume that many of the features of that mathematics (though we cannot really say just which ones) will reflect the way in which those mathematicians developed and practiced their craft (though we cannot really say just which ones). We will also expect that the mathematics that culture has developed will have something to do with the kinds of activities that the society conducts, and with

9 We do not patronisingly suppose that other societies are only capable of contriving basic mathematics, but only that many of them will lack the large guild of full time mathematicians that features in 'our' society. It is not a question whether other societies are 'capable' enough to develop 'our' mathematics - but whether they see the need to develop it. For example, Gay and Cole (1967) remark that among the Kpelle there "are few occasions for counting beyond approximately 30 or 40" (p.42). Similarly, the upshot of Saxe's studies (e.g., Saxe, 1982; Saxe and Moylan, 1982) of the Oksapmin's number use seems to be that their body-counting system up to numbers of twenty-seven was appropriate for all practical purposes of their everyday life. However, with the introduction of currency by the colonialists their mathematical system was bound to change. 
the kinds of circumstances the society provides for their work, the occasions for calculating, working out, formalizing, etc. (but again we cannot really say which ones).

\section{Counting and mathematical-philosophical theories of}

\section{counting}

Within relativistic arguments the examples are typically taken from basic mathematics, in particular elementary arithmetic (what is often called 'numeracy'). However, despite (or perhaps due to) the basic nature of these examples, one needs to be careful to make explicit the level in which they are described by the analyst. We want to distinguish between the everyday practical activities of counting using numbers (e.g., when buying goods, looking at a bank account, etc.) and mathematicalphilosophical accounts of counting and number.

We will take Verran's study of Yoruba numbers (in Watson, 1990) as an example ${ }^{10}$. Verran notes that natural numbers are among "the most sacred Platonic objects” (p. 284) and uses Zermelo and von Neumann's metamathematical-philosophical accounts of number to compare English and Yoruba numbers (p. 307). For us Verran’s project thereby commits a category mistake by conflating the common-sense with the theoretical-philosophical level.

${ }^{10}$ In a later book (Verran, 2001), she repudiates her earlier relativist project. We will deal with Verran's post-relativist project (our term) in a separate paper. Here, we only want to mention that even in her later work she continues to theoretically-ontologically redescribe mundane practices. For example, the example of Mr. Ojo is from her later not her earlier work. We think a happier way forward is ethnomethodological descriptions of practice (see, e.g., Macbeth, 2002). 
We question whether a mathematical-philosophical schematization could ever capture the sense that everyday practical numeracy has. In our view, formal mathematical theories are not meant to be a model or representation of our everyday numerical practices. In other words, people can engage in counting and calculating even if they do not know any formal mathematical-philosophical theories about mathematics. Put differently:

- $\quad 0,1,2,3,4,5,6,7,8,9,10, \ldots$ and

- $\quad 0, \mathrm{~s}(0), \mathrm{s}(\mathrm{s}(0)), \mathrm{s}(\mathrm{s}(\mathrm{s}(0))), \mathrm{s}(\mathrm{s}(\mathrm{s}(\mathrm{s}(0)))), \ldots$

are not on the same footing (although the second schematizes the same numerical progression as the first), but are operating at the different levels of everyday practicality on the one hand, and that of technical mathematics on the other. Dedekind, in his mathematical-philosophical discussions of number in The nature and meaning of numbers (1888), seems to have been aware of this:

This memoir can be understood by any one possessing what is usually called good common sense; no technical philosophic, or mathematical, knowledge is in the least degree required. But I feel conscious that many a reader will scarcely recognise in the shadowy forms which I bring before him his numbers which all his life long have accompanied him as faithful and familiar friends; [...]. (Dedekind, 1909 [1888], p.33, our emphasis)

We therefore argue that there is a crucial difference between everyday counting, adding, multiplying, etc - and technical mathematical-philosophical accounts of the form and structure of number schemes. Those who have mastered basic numeracy do so without familiarity with or dependence on formal theories of mathematics. cultural comparison. Journal for the Theory of Social Behaviour 36 (2), 97-117. 
This distinction between mundane practices and philosophical-scientific theories about that practice would also apply, say, in physics. The distinction would be between 'an apple falling from a tree' and a Cartesian, Aristotelian, Newtonian, or Einsteinian physical account of this event. As Triplett (1986, p.447) notes:

we can know an ordinary observational event - that, say, the apple fell from the tree - without knowing the underlying Newtonian physics that explains how and why it fell. We can imagine disputes in pre-Newtonian days between, say, Cartesian and Aristotelian theoretical accounts of why the apple fell. Indeed we can imagine societies in which no general physical explanation is offered. But we are not thereby inclined to conclude that observers in those societies have no knowledge that the apple fell from the tree.

So why do relativists such as Verran invoke philosophers of science and mathematics in discussing rather ordinary or basic examples? In our view this is because the (supposed) differences are not to be found on the (observable) practical level, but on the (unobservable) theoretical-ontological level. The kind of difference that Verran is after, is not to be found on the practical level (e.g., in the difference between the imperial and metric way of measurement). As she herself notes:

mathematically, Yoruba quantification and modern Indo-European derived quantification were the same (Verran, 2000b, p.356).

So more is required to give life to claims about the relativity of number. That 'more' comes in the assumption of a folk psychology on behalf of people, i.e., in adopting a Whorfian account of language. Relativists make the ubiquitous cognitive supposition that thinking is theorizing, i.e., that in order to be able to use language, people need a theory (an ontology or a worldview) of the constituents of the world. In Verran's case, she assumes that being able to count, presupposes an ontology of number. cultural comparison. Journal for the Theory of Social Behaviour 36 (2), 97-117. 
Verran further stipulates that the form of such an ontology of number will be that traditional (Western) metaphysics ${ }^{11}$. This is reflected in Verran's use of different philosophical accounts of numbers to investigate the number use in English and Yoruba respectively (rather than trying to show that there are practical differences in calculation in the course of conducting everyday affairs). For Verran there seems to be no question that some philosophical account of number will be the correct one; the question is only which one. She argues:

As an English-language user of number, von Neuman's [sic] account seems to me to be intuitively correct. [...] In contrast, I suggest that a Yoruba speaker would choose Zermelo's account of number as correct. (Watson, 1990, p.307)

In contrast, following Wittgenstein we argue that neither is correct, because correctness it not at issue here. As we have argued above, both von Neumann's and Zermelo's accounts of number are not on the same level as our practical grasp of numbers. Furthermore, both von Neumann's and Zermelo's accounts are representations of the same number system, 'our' standard Western arithmetic ${ }^{12}$, and are therefore mathematically equivalent in central respects. There is simply no reason why one should think that one of them best formulated 'our practice'.

\footnotetext{
${ }^{11}$ One of the ironies in some relativistic texts is that the arguments, despite their emphasis on the conceptual autonomy of non-Western cultures insist on construing those in terms of the traditional preoccupations of Western metaphysics.

12 It seems like an incredible coincidence that Zermelo, who presumably never even heard of the Yoruba, unwittingly formulated a set-theoretical account of number that captured the Yoruba's concept of numbers.
} 
Verran provides no mathematical or anthropological explanation as to how either von Neumann's or Zermelo’s account could be taken by an English or Yoruba speaker as a better or more intuitive formulation of the number systems being used. Verran thus engages in what we call the 'ontologising of mundane phenomena' (for a critique along similar lines see Lynch, 1995).

We think that our view is supported by Quine (whom Verran explicitly draws upon):

Actually there has been no dispute that I know of over the relative intuitiveness of the two versions. One uses Frege's version or von Neumann's or yet another, such as Zermelo's, opportunistically to suit the job at hand, if the job is one that calls for providing a version of number at all. [...] Each of the three progressions or any other will do the work of natural numbers, and each happens to be geared also to further jobs to which the others are not. (Quine, 1960, p.263)

In other words, a disagreement among mathematicians or philosophers about the nature of number does not imply a disagreement in the actual counting practices. Von Neumann and Zermelo do not quarrel whether $7+5=12$, but how we should (mathematically and/or philosophically) conceptualize this equation. Verran assumes, in a regressively foundationalist manner, that philosophy underpins practices.

For the relativist, philosophical representations of counting practices are what might appropriately portray users' understanding of, for example, elementary arithmetic. Our reaction to this assumption is to wonder: Is it not surprising that such ontological differences make no detectable difference to the results of enumeration and computation? That is to say, the results of the various calculations are the same and do not show up in intra-cultural contacts. It might be that cultures use different methods cultural comparison. Journal for the Theory of Social Behaviour 36 (2), 97-117. 
to arrive at these results - but that is not the kind of difference that Verran is arguing for (and would hardly be a significant difference). If the relativist does not stipulate some kind of ontology on behalf of actors, the relativistic enterprise does not get off the ground.

\section{Calculation within different mathematical systems}

Let us look at another example, developed by Bloor $(1973,1976)$ and in defense of him elaborated by Jennings (1988), namely the possibility of different arithmetical systems. Bloor and Jennings want to challenge the idea that ' $5+7=12$ ' is a necessary mathematical truth. As in the previous case, on closer inspection of the offered materials it turns out that they are a collection of red herrings. Furthermore, what is ostensibly persuasive about the materials shows that the biggest red herring of all is the idea that a sociology or an anthropology is needed here, i.e., that anything in addition to clarity about the mathematical examples is involved.

What is presented as evidence consists in nothing but what is an elementary and familiar mathematical fact, namely that one can construct arithmetical systems on different 'bases', i.e., rather than counting up to ten one could instead count 'up to eight', e.g.: $0,1,2,3,4,5,6,7,10,11,12,13, \ldots$ (and hence $5+7$ would be 14 ). In principle, there is no limit to the bases that one could choose to use - or even no limit to systems without any base (which would then be finite, say up to 27 or 134). As we have outlined above, 'our' standardized arithmetic is part of the practical affairs of our society and hence widespread. It is what children are taught very early in life (and not only in school). It is what most of us, knowing little else about mathematics, might take mathematics to be. However, even within our practical life there are places cultural comparison. Journal for the Theory of Social Behaviour 36 (2), 97-117. 
where other kinds of computational practices are employed, e.g., days of the week (base 7), hours of the days (base 12 or base 24) - and even non-base systems (the alphabet).

Pointing to another culture where people calculate on another base does not mean that 'their' mathematics stands in opposition to 'our' mathematics (any more than their cuisine is 'in opposition' with ours, or their legal system at odds with ours - though they might come into conflict in the context of, say, an imperialist bid to homogenize practice across previously independent communities; but that is neither an epistemological point nor one with epistemological import). Of course, there is a difference between the two mathematics: in the everyday practice of our society, calculation according to base 10 is heavily standardized (but not utterly ubiquitous), whereas in their society calculation according to a different base is practiced. It is not, however, as though mathematics (any 'our' is gratuitous at this point) insists that calculation to base 10 is the only possible form of calculation (see our points above). Hence different bases do not stand in mathematical 'conflict' with another.

Jennings (1988, p. 100), taking the example of $5+7=12$ argues

Now it is not very difficult to imagine a culture in which $[5+7=$ 12] is regarded as false - we can imagine a culture in which $5+7=$ 14 , or another in which $5+7=10$.

Jennings here seems to suggest that $5+7=12$ is something that we 'believe' in (as he puts it: “regarded as false”). However, as we have argued above, arithmetical statements are not 'believed' (in contrast to, say, a belief in God or in the strength of a football team). 
To avoid confusion we need to distinguish between conceiving of a statement as an expression 'of and in' the system or as an expression 'about' the system. In other words, one can make a mistake within the system (e.g., by claiming that ' $5+6=12$ '), but the system itself cannot be false (or true) since it provides the standards for correctness. The same applies, for example, to the rules of the road: one can drive on the wrong side in England (i.e., on the right) - but the English driving rules cannot be wrong in and of themselves. Furthermore, as Shanker (1987) notes:

Even a primitive counting system - e.g. Wittgenstein’s '1, 2, 3, 4, 5 many' example where ' $3+4=4+5$ ' - is not 'wrong' compared to a sophisticated method; it is simply designed to meet different needs; which presumably it satisfies. (pp.298-299) ${ }^{13}$

It would be wrong to suppose that the use of a different base shows that ' $5+7=12$ ' is false, for it would be to suppose that the expressions in the different systems are other than superficially alike. The fact that one would not get 12 as the sum of $5+7$ when adding on a different base than 10 does not show that, calculated on base 10 , the original addition is false. It is correct if one is counting to base 10. The operation of adding 5 to 7 on another base is a different operation than that involved in adding them on base 10. Further, the two different ways of calculating are not in any mathematical conflict with each other, as they are, mathematically, isomorphic: one can show that the $5+7=20$ (base 6 ) is the equivalent of $5+7=12$ (base 10 ).

\footnotetext{
${ }^{13}$ Note that 'many' here is not quite the same as 7 or 9 , but more like 'over 5' (and both $3+4$ and $4+5$ are 'over five'). Consequently, 'equals' does not play quite the same role here.
}

Greiffenhagen, C. and W. Sharrock (2006). Mathematical relativism: logic, grammar, and arithmetic in cultural comparison. Journal for the Theory of Social Behaviour 36 (2), 97-117. 
Jennings is treating ' $5+7$ ' as though it were an identical arithmetical expression in two discrete systems, suggesting that someone who calculates on one base is denying the correctness of computations made on a different base. But here is where the fact that computation is relative to a system bites back against Jennings. ' $5+7$ ' is not the same proposition in the two different systems, and so even if two propositions of superficial similarity have different values in the two systems, it does not show that one affirms what the other denies. Unless we follow logical positivists and stipulate that '(mathematical) marks on the paper' (such as ' $5+7=12$ ') are part of a neutral or objective language, then ' $5+7=12$ ' is neither right or wrong without specifying which system it belongs to. The expression ' $5+7=12$ ' has no sense independently of one system or another. Without knowing which arrangement it belongs to, one has no means of deciding whether it is right or wrong. In other words, not just the expression but also rightness and wrongness belong to and get determinate sense and application from a system.

In sum, when imagining two cultures, one in which ' $5+7=12$ ' and one in which ' $5+$ $7=14$ ', we have two points to bear in mind: firstly, the different systems are (with possibly a bit of work) intelligible as mathematically equivalent, and, secondly, the ' 5 ' in the first equation and the ' 5 ' in the second equation, although of the same graphical shape, play different roles in the two systems.

In sum, our arguments pertain to the reciprocal intelligibility of mathematical expressions in different cultures. For example, it is a commonplace that the Romans would not immediately be able to understand 'our' mathematics. Without prior instruction in, most importantly, our Arabic numerals, an expression such as ' $2+3=$ cultural comparison. Journal for the Theory of Social Behaviour 36 (2), 97-117. 
5' would to a Roman at first seem more like a drawing than a mathematical statement.

A Roman would simply not be able to read the signs. However, firstly, a Roman could understand and approve the 'translation' into Roman numerals ('II + III = V'), and, secondly, with very little instruction a Roman who was already able to calculate (with Roman numerals) could be brought up to speed in our mathematics.

It is easy to forget that many of the purportedly different systems are in fact, from a mathematical point of view, equivalent, and can be coordinated with each other (in the way that imperial and metric measurement are different but equivalent and can be coordinated, so that expressions in one can be converted into expressions in the other). This is of course not to deny that one form might not be more practical for specific purposes.

\section{Do we have different numbers than the Ancient Greeks?}

As a final example, we will look at disagreements among Ancient Greek mathematicians and philosophers as to whether 'one' is a number or not. This example is given by Bloor (1976, Chapter 6) as an example of an 'alternative' mathematics:

Is 'one' a number? The following statements were commonplace in early Greek mathematics: one is not a number; one is neither odd nor even but even-odd; two is not an even number. Nowadays each of these claims is rejected as false. For us, one is a number just like any other. Frege could cite it as such in his arguments without a second thought. (Bloor, 1976, p. 98; emphasis in original)

Jennings (1988, p.97) uses this example to show that Bloor does, contra his critics (e.g., Triplett, 1986, 1988, 1994), demonstrate that an alternative mathematics in a cultural comparison. Journal for the Theory of Social Behaviour 36 (2), 97-117. 
strong conflictual sense is possible. Bloor and Jennings are satisfied that this example would demonstrate that 'alternate mathematics' could exist, because it seems to imply that the Ancient Greek mathematicians did not even agree with us as to something as basic as what a 'number' is. However, as noted in the introduction, we need to be careful to see the nature and extent of the supposed difference between the Greeks and ourselves with respect to number.

We do not deny that some Greek philosophers or mathematicians wondered whether one was a number. However, they did not all agree that it was not. To the extent that some Greek mathematicians held that one was not a number, then we might say, that the Greek mathematicians had a different concept of number than we do. But beware! Their argument was not about whether Zeus was a number or whether a pig's ear was a number. It was a disagreement about something that they recognized as being very similar to the other 'numbers' (two, three, four, and so on). Further, it was not a disagreement which involved any attempt to exclude one from calculation, i.e., from use in conjunction with other numbers to do sums in everyday life. Nor was it a disagreement whether one was a number, and whether two was a number, and whether three was a number, and whether four was a number - it was about one specifically (and possibly also two).

Furthermore, there was not any question with respect to everyday numeracy as to whether it was possible for someone to own only 'one' sheep or have only 'one' coin left in their purse, or to sell off one of their two sheep leaving them with only 'one'. Most computation practiced in Ancient Greece was independent of these considerations. In other words, whose concept of number are we are talking about: cultural comparison. Journal for the Theory of Social Behaviour 36 (2), 97-117. 
the concept of all the people living in Greece at that time (or all of us?), including those that were barely able to calculate - or only of those very few that were part of what we might call professional mathematics? Would Bloor or Jennings want to say that shopkeepers' concepts of number were very different in Ancient Greece from those current in Modern England?

With respect to the rest of the natural numbers in particular and arithmetic in general, the Greek mathematicians had very much the same concept of number as we do - to the extent that many of their arithmetical proofs are preserved in our mathematics. Furthermore, as Bloor (1976, p.98) himself notes, the reason that for some Greek mathematicians one is not a number is "because they saw it as the starting point or generator of number”. In other words, the Greek mathematicians disagreed about the status of one relative to the rest of the number system for the same reasons that modern mathematicians often give one a special place in the number system: with one, it is possible to generate all the other numbers (i.e., 2 is $1+1,3=2+1=(1+1)$ +1 , and so on); furthermore, one is not treated as a prime number (although one is only divisible by itself).

It were these distinctive (mathematical) features of one that gave rise to the Ancient Greek’s discussion as to whether it was to be regarded as just one more number amongst the others, or whether it was so special it was not to be included within the number series - just as zero sometimes is and sometimes is not so included as a cultural comparison. Journal for the Theory of Social Behaviour 36 (2), 97-117. 
natural number in modern mathematics ${ }^{14}$. In fact, had Bloor based his account not on Frege but on Husserl (both $19^{\text {th }}$ century German philosophers) he would have had to argue that the Greeks had the same concept of number, as for Husserl zero and one were not numbers but only number-like:

there is something number-like about 'one' and 'none' in the sense that these words can also serve in certain contexts to answer the question 'how many'. Nonetheless one and none are not numbers in the original sense, according to Husserl; they are not Anzahlen. (Miller, 1982, p.123)

Our argument does not deny a difference between those who denied one as being a number and our mathematics, nor do we deny that on that basis Ancient Greek mathematics could be called an alternative mathematics. We only would like to note that on this basis it does not seem to take very much to qualify something as an alternative mathematics. In other words, one could equally well deem the differences over one as so insignificant that it would be an overstatement to count this as a case of an alternative mathematics. One might equally well say that the Ancient Greeks had much the same concept of the natural numbers as we do - save for some intelligible and computationally inconsequential issues about the status of one relative to the rest of the number sequence (as, we repeat, it did not mean that anyone who counted their sheep in the Greek’s arithmetic would always be one sheep short).

\footnotetext{
${ }^{14}$ It's a bit like arguing about whether the foundations are part of the house or not - which does not entail any disagreements over which part of the construction is the foundations and which 'the rest of the house'. It is also reminiscent of Wittgenstein's arguments about the 'standard metre' as being neither one metre long or not one metre long in an attempt to emphasise its special role in the metric system as the thing that is used to give definitive measurement, not something itself to be measured. This is not an argument that the standard metre has no length.
}

Greiffenhagen, C. and W. Sharrock (2006). Mathematical relativism: logic, grammar, and arithmetic in cultural comparison. Journal for the Theory of Social Behaviour 36 (2), 97-117. 
Overwhelmingly the Ancient Greeks had the same concept of number as we do, which is why their mathematics is treated as historically continuous with 'our' mathematics, e.g., why our mathematicians continue to accept many of their proofs (although they might be reformulated in modern mathematical notations and forms). The mathematician Hardy, for example, emphasizes that modern mathematicians can understand Greek mathematics:

The Greeks were the first mathematicians who are still 'real' to us to-day. Oriental mathematics may be an interesting curiosity but Greek mathematics is the real thing. The Greeks first spoke a language which modern mathematicians can understand; [...] So Greek mathematics is 'permanent', more permanent even than Greek literature. Archimedes will be remembered when Aeschylus is forgotten, because languages die and mathematical ideas do not. (Hardy, 1940, pp.20-21)

In other words, the relativist who argues that the Greek and our mathematics constitute 'alternate' mathematics, cannot account for the continuous nature of mathematics ${ }^{15}$.

To repeat the point that we made earlier: the philosophical-theoretical status of the number one does not underpin the ordinary practices of computation, for, as noted, nothing in everyday computational practice is changed by adopting either one or other

\footnotetext{
${ }^{15}$ Hardy also notes that Oriental mathematics differs more significantly from 'our' mathematics than does the Greeks'. However, this is no embarrassment for our argument, since it takes as a given for all parties to discussions about the 'relativity' of mathematics that there is no a priori limit to the variation that there can be between the mathematics of one culture and another, leaving us free to argue that the kind of construal that the relativist would lay on these variations is implausible. Our point is about the intelligibility of claims that alien mathematics could directly contradict each other and yet both be true, and we have tried to show that the cases which relativists offer do not intimate any such possibility,
} 
side on such a point. We thus agree with Freudenthal (1979, p.75) who argues that Bloor and Jennings fail "to distinguish between meta-mathematical arguments and mathematical proofs”. As Triplett (1986, p. 445) points out: Bloor's and Jennings’ arguments do not pertain to mathematics, but to, at best, metamathematics or "various philosophical theories about mathematics”16.

\section{Conclusion: theoretically corrupted portrayals of practice}

The idea that mathematical equations constitute empirical propositions or contain ontological assumptions (rather than express rules of a system) runs through much of relativists' discussions. This leads the relativist to the supposition that it is necessary to investigate what the mathematical practices stand for, either by looking at facts of nature or at a people's concepts of numbers. Consequently, our anti-relativist arguments advanced in this paper are not to be understood as a (realist) defense of Western science (as, for example, pursued by so many of the opponents to the Sociology of Scientific Knowledge and poststructuralist approaches). Instead, they form an anti-intellectualist Wittgensteinian interrogation of the idea that cultural practices are theory-dependent.

The fact that practices of arithmetical computation are predominantly constant or equivalent across the different examples is not actually denied by the relativist, but is left in the background in the search (by those desperately seeking differentiation) for

\footnotetext{
${ }^{16}$ Bloor and Jennings may be right to argue that the Ancient Greeks may not have distinguished between philosophy and mathematics, or between mathematics and meta-mathematics, but the point of distinguishing these in such a connection has less to do with correctly characterising Greek practice than it does with conveying to contemporary readers a sense of how significant a difference there is between our practice and theirs with respect to e.g. numeracy.
} 
something, anything, in the practice of calculation that might be considered a startling difference from our practice. For the relativist it is as though thinly described, inconsequential, or peripheral instances could go proxy for proper evidence to support the claim that there is a radically alternative mathematics - a mathematics which would exhibit a discrepant way of thinking to our own and that would come into irresoluble conflict with our mathematics.

We have been trying to suggest that relativists are apt to confuse the rigidity of application of the rules that make up arithmetical systems (i.e., that they are to be applied everywhere the same, regardless of who is doing the calculation) with people's supposed beliefs about the status of mathematical truths (e.g., that there can only be one mathematical notation). Consequently, relativists are apt to consider it a deep and troubling finding that the range of specific mathematical systems is possibly restricted to a particular culture, as for the relativist this seems to imply that thereby the rigidity of those rules is also restricted. However, that there can be different mathematics from our standard mathematics is not something that ordinary people are practically unaware of. In fact, we are all familiar with instances where, for one example, five plus eight equals one (on a twelve hour clock). We are thus not denying that there are different mathematics, for there are lots of different mathematical systems, and they differ in all sorts of ways. Everyone familiar with more than elementary mathematics understands that. We are only contesting the relativist's idea that these are radical divergences, entailing ontological diversity between cultures, and that the manifest equivalences between mathematics are only superficial, i.e., disguise the real and deeper differences underpinning them. cultural comparison. Journal for the Theory of Social Behaviour 36 (2), 97-117. 
On closer inspection, the offered examples turn out to depend on theoretically corrupted portrayals of practice on one or both sides of the cultural separation (and of a conflation of philosophical theories about, e.g., measuring with the mundane practice of measuring). The supposedly significantly alternative mathematics described turn out to involve minor disputes within the philosophy of mathematics (e.g., whether one is a number or not) with little practical consequences. Or they depend on trying to insinuate some philosophical preconceptions about the nature of mathematics into everyday understandings of computation on the assumption that some such preconceptions must be integrally indispensable to the use of the number system.

\section{Acknowledgements}

Wes Sharrock would like to thank the Arts and Humanities Research Board (AHRB) for an Innovation Award (grant number B/IA/AN10985/APN17690) to support the work reported in this paper. We would like to thank Mike Lynch and two anonymous reviewers for comments on an earlier draft.

\section{References}

Baker, G. P. and P. M. S. Hacker (1985). Wittgenstein: Rules, Grammar, and Necessity. Oxford: Blackwell.

Bloor, D. (1973). Wittgenstein and Mannheim on the sociology of mathematics. Studies in History and Philosophy of Science 4 (2), 173-191.

Bloor, D. (1976). Knowledge and Social Imagery. London: Routledge \& Kegan Paul.

Bloor, D. (1994). What can the sociologist of knowledge say about $2+2=4$ ? In P. Ernest (Ed.), Mathematics, Education and Philosophy: An International Perspective, pp. 21-32. London: Falmer.

Dedekind, R. (1909). The nature and meaning of numbers. In Essays on the Theory of Numbers (Translated by W. W. Beman), pp. 29-115. Chicago: Open Court. Translation of 'Was sind und was sollen die Zahlen?' (1888). 
Freudenthal, G. (1979). How strong is Dr. Bloor's 'strong programme'? Studies in History and Philosophy of Science 10 (1): 67-83.

Gay, J. and M. Cole (1967). The New Mathematics and An Old Culture: A Study of Learning among the Kpelle of Liberia. New York: Holt, Rinehart and Winston.

Gerrard, S. (1991). Wittgenstein's philosophies of mathematics. Synthese 87 (1), 125-142.

Greiffenhagen, C. (forthcoming). Gerrymandering the formal-informal relationship: manufacturing an opposition between 'official' and 'unofficial' mathematics.

Greiffenhagen, C. and W. Sharrock (forthcoming a). Logical relativism.

Greiffenhagen, C. and W. Sharrock (forthcoming b). Linguistic relativism.

Hardy, G. H. (1940). A Mathematician’s Apology. Cambridge: Cambridge University Press.

Jennings, R. C. (1988). Alternative mathematics and the strong programme: Reply to Triplett. Inquiry 31 (1): 93-101.

Lave, J. (1988). Cognition in Practice: Mind, Mathematics, and Culture in Everyday Life. Cambridge: Cambridge University Press.

Louch, A. R. (2000 [1966]). Explanations and Human Action. Christchurch, New Zealand: Cybereditions. Originally published 1966, Oxford: Basil Blackwell.

Lynch, M. (1995). The idylls of the academy. Social Studies of Science 25 (1): 582-600.

Macbeth, D. H. (2002). A commentary on instructional design. Journal of the Learning Sciences 11 (2\&3): 373-386.

Miller, J. P. (1982). Numbers in Presence and Absence: A Study of Husserl's Philosophy of Mathematics. The Hague: Martinus Nijhoff.

Quine, W. V. O. (1960). Word \& Object. Cambridge, MA: MIT Press.

Raven, D. (1996). The enculturation of logical practice. Configurations 4 (3): 381-425.

Reed, H. J. and J. Lave (1979). Arithmetic as a tool for investigating relations between culture and cognition. American Ethnologist 6 (3): 568-582.

Saxe, G. B. (1982). Developing forms of arithmetical thought among the Oksapmin of Papua New Guinea. Developmental Psychology 18 (4): 583-594.

Saxe, G. B. and T. Moylan (1982). The development of measurement operations among the Oksapmin of Papua New Guinea. Child Development 53 (5): 1242-1248.

Shanker, S. G. (1987). AI at the crossroads. In B. P. Bloomfield (Ed.), The Question of Artificial Intelligence: Philosophical and Sociological Perspectives, pp. 1-58. London: Croom Helm.

Triplett, T. (1986). Relativism and the sociology of mathematics: Remarks on Bloor, Flew, and Frege. Inquiry 29 (4): 439-450.

Triplett, T. (1988). Azande logic versus Western logic? The British Journal for the Philosophy of Science 39 (3), 361-366.

Triplett, T. (1994). Is there anthropological evidence that logic is culturally relative?: Remarks on Bloor, Jennings, and Evans-Pritchard. The British Journal for the Philosophy of Science 45 (2), 749-760.

Verran, H. (2000a). Logics and mathematics: challenges arising in working across cultures. In H. Selin (Ed.), Mathematics Across Cultures: The History of non-Western Mathematics, pp. 55-78. Dordrecht: Kluwer. 
Verran, H. (2000b). Accounting mathematics in West Africa: some stories of Yoruba number. In H. Selin (Ed.), Mathematics Across Cultures: The History of non-Western Mathematics, pp. 345-371. Dordrecht: Kluwer.

Verran, H. (2001). Science and an African Logic. Chicago: University of Chicago Press.

Watson, H. (1987). Learning to apply numbers to nature: a comparison of English speaking and Yoruba speaking children learning to quantify. Educational Studies in Mathematics 18 (4): 339-357.

Watson, H. (1990). Investigating the social foundations of mathematics: natural number in culturally diverse forms of life. Social Studies of Science 20 (2): 283-312.

Wittgenstein, L. (1953). Philosophical Investigations (Translated by G.E.M. Anscombe). Oxford: Basil Blackwell.

Wittgenstein, L. (1976). Wittgenstein's Lectures on the Foundations of Mathematics, Cambridge, 1939 (Edited by C. Diamond). Chicago: University of Chicago Press.

Wittgenstein, L. (1978). Remarks on the Foundations of Mathematics (Edited by G. H. von Wright, R. Rhees and G. E. M. Anscombe. Translated by G. E. M. Anscombe) (Third ed.). Oxford: Basil Blackwell. 